\title{
Inhibition of intraspecific aggression in male hamsters by septal stimulation
}

\author{
M. POTEGAL, A. BLAU, and M. GLUSMAN \\ New York State Psychiatric Institute, New York, New York 10032
}

\begin{abstract}
Male hamsters selected for intraspecific aggressiveness were implanted with a movable monopolar electrode that was lowered through the septal region. At current levels which did not produce any motor automatisms or stereotyped competing responses, septal region stimulation prevented the initiation of subjects' attacks on muzzled and analgesic-treated target hamsters. Thresholds for this aggression inhibition appeared to reach a minimum in the midand ventral septum. Stimulation at current levels necessary for aggression inhibition had no effect on sunflower seed acceptance. In operant tests, the reinforcing value of the aggressioninhibitory stimulation appeared to be positive at low current levels and negative at high levels.
\end{abstract}

Stimulation of the septal region inhibits interspecific or predatory aggression in rats and cats (Potegal, Gibbons, \& Glusman, 1980; Siegel \& Skog, 1970) and defensive aggression in rats, cats, and monkeys (Brayley \& Albert, 1977; Rubinstein \& Delgado, 1963; Sierra, Acuna, Otero, \& Dominguez, 1972). However, the effects of septal stimulation on intraspecific aggression have not been well established. We chose the hamster for this study of the role of the septum in intraspecific aggression because intraspecific aggression is relatively easy to obtain in this species but does not lead to the excessive physical damage that may occur with the larger species. Furthermore, a number of studies have shown that, in the hamster, unlike the rat, septal lesions routinely increase intraspecific aggression (Janzen \& Bunnell, 1976; Johnson, Poplawsky, \& Bieliauskas, 1972; Potegal, Blau, \& Glusman, 1981; Sodetz \& Bunnell, 1970; but see Shipley \& Kolb, 1977).

To insure that we would be testing the effects of septal stimulation on subjects' initiations of attack rather than on their responses to being attacked, it was necessary to insure that the implanted subjects would be the aggressors in any encounter. To this end, we used a technique we had previously developed in which active, "spontaneously" aggressive subjects are presented with nonaggressive, muzzled, and drugtreated targets. With diminished and relatively invariant spontaneous activity and responsivity to attack, these target hamsters provide a standardized situation in which the subject's initiation of aggressive behavior can be measured (Potegal, Blau, Black, \& Glusman, 1980).

Study of the literature cited in the first paragraph clearly indicates that septal stimulation may inhibit

We would like to thank Ludmila Skaredoff for preparation of the histological material. This work was supported by Grant RO 3 MH 28836-01 from the National Institute of Mental Health and by a grant from the Harry F. Guggenheim Foundation. aggression for a number of reasons: It may evoke competing motor responses or it may have primary rewarding and/or aversive effects that produce aggression inhibition as a secondary consequence of stimulation. Alternatively, it may produce a generalized inhibition of behavior. Thus, we decided to test any inhibitory sites we found for such effects. To maximize both our chances of finding inhibitory sites and our ability to map their distribution within an individual subject, we elected to use a movable electrode.

\section{METHODS}

\section{Selection of Aggressive Subjects}

Upon receipt from the breeding farm, adult, male outbred Syrian hamsters (3-4 months old) were isolated in $51 \times 41 \times 20 \mathrm{~cm}$ transparent plastic cages with a woodchip substrate and ad-lib food and water. They were maintained on a reverse 14-hr-dark/ 10-hr-light cycle. No sooner than 2 weeks after being placed under these conditions, the subjects were screened for aggressiveness by having a muzzled, methotrimeprazine-treated target hamster (see below) placed in their home cage for $10 \mathrm{~min}$ on two occasions. Eleven animals that attacked the target on at least one occasion (approximately $20 \%$ of the population screened) were selected as subjects. For this screening, and for the rest of this study, an attack was defined as a bite delivered from a full aggressive posture (Grant \& Mackintosh, 1963) which caused the target to squeal, struggle, and/or assume the on-back posture (Floody \& Pfaff, 1977). Because the targets' locomotion and responsivity were reduced by the drug, attacks launched from the full aggressive posture were more common than the sideways posture attacks and the chase-follow sequences usually observed in unrestrained encounters (Potegal et al., 1980).

Electrode Manufacture, Implantation, and Stimulation Parameters

The subjects were anesthetized with $62.5 \mathrm{mg} / \mathrm{kg}$ ip sodium thiopental (Pentothal) and stereotaxically implanted with a movable electrode. This electrode was a .25-mm Formvar-coated stainless steel wire mounted in a 0-80 set screw. The electrodebearing set screw was threaded into a 6-mm length of 0-80 internally tapped 16-ga tubing mounted in an insulating Teflon cup. Tissue damage caused by eccentric rotation of the electrode was reduced by a $.33-\mathrm{mm}$ guide hole in the Teflon cup. This electrode assembly was attached to the skull by 000 self-tapping screws and a spring-clip skull anchor. Electrical connection to the 
stimulator was made through a Plastic Products MS 303/2 (untwisted) bipolar electrode, one side of which was soldered to the tubing and the other side of which was connected to the springclip skull anchor. When the implantation was completed, the subjects were given $10 \mathrm{mg} / \mathrm{kg}$ phenoxybenzamine ip to reduce the stress of surgery.

A Grass S4 stimulator, set at 100 pps with a $1-\mathrm{msec}$ pulse duration, provided a biphasic stimulus pulse with a zero net coulomb flow. Current output was continuously monitored on an oscilloscope in parallel with the current-limiting $47 \mathrm{k} \Omega$ resistor in series with the subject.

\section{Preparation of Targets}

Animals that did not attack during screening were grouped 3-5/cage to further reduce aggressiveness. When these animals were to be used as targets, they were treated with $6 \mathrm{mg} / \mathrm{kg}$ im of the nonnarcotic analgesic methotrimeprazine (Levoprome, Lederle) 30-45 min before a session. Treated targets were also muzzled to prevent "retaliatory" bites. We have previously found that, as long as session durations do not exceed $45 \mathrm{~min}$, targets spend most of their time standing motionless. Target pain thresholds remain elevated throughout the encounter by the methotrimeprazine (Potegal et al., 1980).

\section{Behavioral Testing}

Behavioral testing was done in the subjects' home cages, which fitted inside the testing chamber housing the Merotac $\mathrm{Hg}$ commutator and custom-made Plastic Products cable. At each level of electrode descent, the following screening procedure was used: a water dish, food, and a cork for gnawing and carrying were placed inside the cage. The animals were then stimulated at increasing current levels until a consistent motor or behavioral response appeared. No sooner than $24 \mathrm{~h}$ after a motor threshold was established, aggression-inhibitory stimulation (AIS) threshold testing began.

To reduce the amount of stimulation given on any one day, AIS thresholds were determined in two stages. Initial stage (provisional AIS threshold determination): On the first, baseline, trial, a target hamster was placed in the cage next to the subject. The latency to attack the target was established on this baseline trial in the absence of brain stimulation. On the next trial, current at a level just below that producing an overt response was applied $.5 \mathrm{~min}$ before the target was placed in the cage. The current remained on until the subject attacked or the criterion for inhi-
Table 1

Effects of AIS on Mean Attack and Seed Acceptance Latencies

\begin{tabular}{clccccc}
\hline & & \multicolumn{2}{c}{ Attack } & & \multicolumn{2}{c}{ Seed Acceptance } \\
\cline { 3 - 4 } Trial & AIS & Mean & SD & & Mean & SD \\
\hline 1 & No & 67.2 & 55.7 & 6.6 & 3.4 \\
2 & No & 46.6 & 34.0 & & 5.9 & 3.0 \\
3 & Yes & & & 7.4 & 6.9 \\
4 & No & 34.6 & 30.1 & & 4.6 & 2.4 \\
\hline
\end{tabular}

Note-All data are latencies, given in seconds. The Trial 3 attack latencies are replaced by the inhibition criterion (greater than Trial 1 or 2 plus $30 \mathrm{sec}$ or greater than 1 min, whichever is longest).

bition was met. This criterion was the absence of attack in a period $30 \mathrm{sec}$ longer than the attack latency on the baseline trial (or, if the baseline attack latency was $<30 \mathrm{sec}$, the criterion was changed to a 1-min period without attack). If the subject attacked the target, the target was removed but current was left on for a short, variable period to prevent attack from becoming an operant escape from stimulation. If inhibition was obtained, current was lowered on successive trials until a provisional threshold value was approximated (i.e., the lowest value of current giving complete and consistent inhibition). Second stage (final threshold determination): No sooner than $24 \mathrm{~h}$ after the provisional threshold level was determined, final threshold value was established and tests of the effects of this AIS value on sunflower seed acceptance were performed. The target was presented in a set of four trials with a 1-min ITI. If Trial 1 and Trial 2 attack latencies were less than 3 and $2.5 \mathrm{~min}$, respectively, insuring a stable "baseline", for that set, stimulation at the provisional threshold preceded and accompanied target presentation on the third trial of that set. Attack latencies on all trials were recorded. The AIS threshold value was accepted as final if (1) the inhibition criterion was met on the stimulated trial and (2) attack did occur on the fourth (nonstimulated) trial. If inhibition was not obtained on Trial 3, the current value was raised in subsequent trial sets; if attack did not occur on Trial 4 (i.e., a "carryover effect" was observed), the trial set was repeated. Trial sets were given, at a rate of no more than $2 /$ day, until a final threshold value was obtained. Thus, the final AIS threshold values given in the results (Table 1 and Figure 1) satisfy the criteria that (1) they were replicated on 2 different test days and that (2) on the second test day, inhibition was

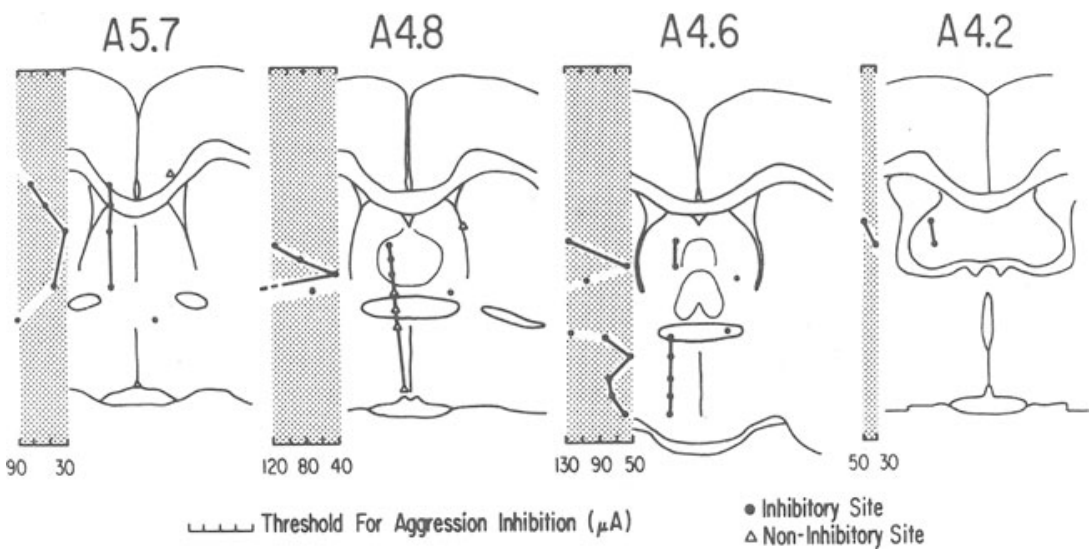

Figure 1. Aggression-inhibitory loci are plotted as filled dots and noninhibitory loci as open triangles on sections redrawn from the Knigge and Joseph (Note 1) atlas (A5.7, A4.8) or interpolated sections $(\mathbf{A} 4.6, \mathbf{A 4 . 2})$. Tracks in which two or more inhibitory loci were found are plotted in the left half of each section; individual loci are plotted in the right half. Each inhibitory locus is represented again by a dot at the same horizontal level within the stippled area immediately to the left of each section. The dots within the stippling indicate the vertical profile of the inhibition threshold. Stimulation at the three ventralmost "noninhibitory" sites in section A4.8 actually facilitated attack. 
found in the third trial of a four-trial sequence in which attack occurred on all other trials. After the final AIS threshold was established, a sunflower seed test was given with the same Trial 3 stimulation structure. Sunflower seed trials consisted of the presentations to the hamster of a sunflower seed wedged in a light spring taped to the end of a long rod. The latency to remove the seed from the spring was recorded.

The reinforcing value of AIS was evaluated in a $20 \times 20 \times 37 \mathrm{~cm}$ chamber with a $5.5 \times 5.5 \mathrm{~cm}$ pedal flush with the floor in one corner. A three-phase automated testing cycle was used: Phases 1 and 3 were baseline, in which pedalpressing had no effect. Phase 2 was a self-stimulation paradigm in which stimulation at AIS threshold values occurred as long as the pedal was depressed. Each phase of the cycle lasted $5 \mathrm{~min}$; the initiation of the next phase was triggered by the pedalpress following termination of the last phase. Total number and duration of pedalpresses were recorded.

In a supplementary test of the effects of AIS on sexual behavior, four subjects were presented with an ovariectomized female which had been brought into estrus by $25 \mu \mathrm{g}$ of estradiol 72 and $48 \mathrm{~h}$ pretest and $500 \mu \mathrm{g}$ progesterone $4 \mathrm{~h}$ pretest. The standard "Trial 3 stimulation" paradigm was used. This test was performed near or at the point of lowest AIS threshold in the electrodes' descent. The electrode was dropped at least a full turn $(320 \mu)$ at the end of the last test at a given locus. At least $23 \mathrm{~h}$ elapsed before the next test.

\section{Histological Preparation}

The animals were given an overdose of Nembutal. The electrode was raised to the minimum threshold point and a $2-\mathrm{mA}$ dc current was passed for $20 \mathrm{sec}$ using the electrode as an anode. The iron ions left by this procedure reacted with the potassium ferrocyanide in the perfusion fluid to produce the Prussian blue reaction at that point (Jasper \& Ajmone-Marsan, 1954). Brains were sectioned at $25 \mu$ while frozen and then stained with cresyl violet after mounting.

\section{RESULTS}

\section{Motor Threshold Determination}

The overt effect most frequently elicited by stimulation was a cessation of locomotor activity during which the animal remained motionless in a quadrupedal posture (mean threshold of $123 \mu \mathrm{A}$ at six points). Orienting movements of the head, usually in the vertical plane, were observed at four locations (mean threshold of $97 \mu \mathrm{A}$ ). Digging was elicited at two points, while pouch filling, feces removal, eye blinks, and seizure activity were seen at one point each. There was no consistent effect of stimulation at current levels below $400 \mu \mathrm{A}$ at the remaining points. No consistent association emerged between the anatomical locus of stimulation and the nature of the motor response. Motor thresholds tended to decline as the electrode advanced, but this tendency was not as systematic as the decline in inhibition thresholds.

\section{Effects of Stimulation on Aggressive Behavior}

Inhibition of aggression was found in 9 of the 11 subjects; the threshold for inhibition was below the motor threshold at 16 of the 20 inhibitory sites. Mean attack latencies on stimulated and nonstimulated trials are presented in Table 1. Profiles of the inhibitory thresholds are reconstructed on standard atlas sections (Knigge \& Joseph, Note 1) in Figure 1. In each of four subjects, only one point was studied extensively enough to meet the criteria for inhibition. A characteristic V-shaped profile in three of four sections suggests a threshold minimum around the midand ventral septum. A variety of behaviors occurred during AIS. Approach toward, sniffing, and following of the target, alone or in combination, were seen on 18 occasions. On 3 of these occasions, the subjects also produced a full aggressive posture, but no attack occurred. "Freezing" was seen on 12 occasions, grooming on 10, and flank-marking, digging, and pouch-emptying on 4 occasions each. On $33 \%$ of the four-trial sets (second stage of threshold determination), "carryover" effects occurred; that is, the subject failed to attack on the fourth (nonstimulated) trial after having been inhibited on the third (stimulated) trial.

\section{Effects of Stimulation on Sunflower \\ Seed Acceptance}

At all 20 points at which aggression could be inhibited, a comparison was made between the latency to accept sunflower seeds on the third (stimulated)

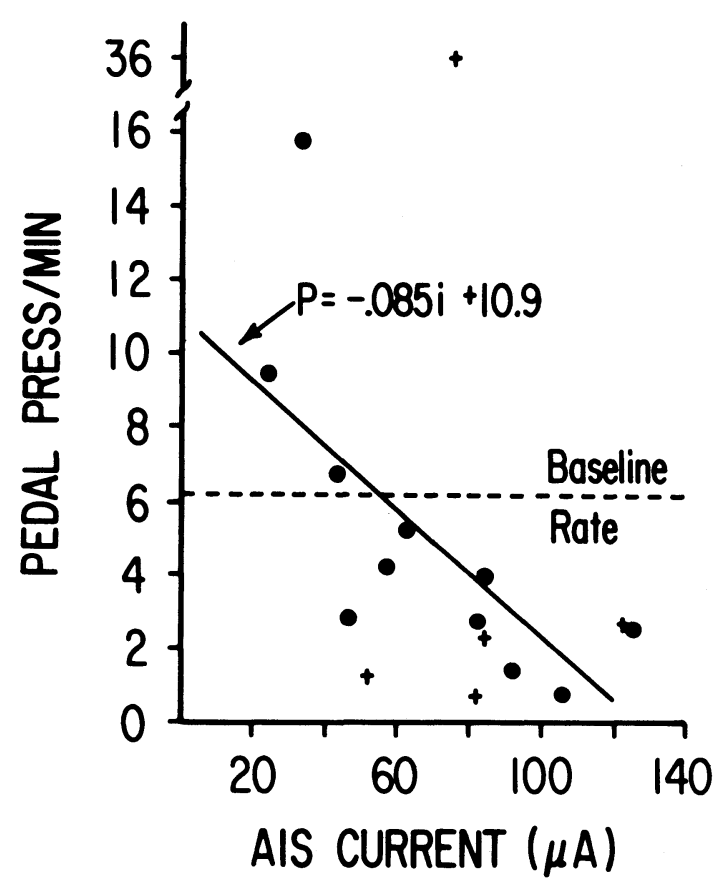

Figure 2. Solid circles are loci dorsal to the anterior commissure; pluses are loci ventral to the commissure (the ventral loci are the ones on the right side of section A5.7 and the left side of A4.6 in Figure 1). The Pearson's $\mathrm{r}$ for the dorsal points is - .652 [t(9) $=2.58, \mathrm{p}<.05$ ), showing a significant negative correlation between pedalpress rate and AIS current intensity. This relationship is not due to different animals' clustering at different ends of the distribution; each of two subjects with three or more inhibitory points in their tracks (left side, sections A5.7 and A4.8, Figure 1) contributed points at both extremes of the distribution. The solid line whose equation is given in the figure is the least squares regression line. Ventral points do not lie along this line. The dotted line is the mean of Phase 1 and Phase 3 baseline (nonstimulated) pedalpress rates. 
trial and the mean of the second and fourth (unstimulated) trials, using a $t$ test for related measures. Stimulation that was effective in completely suppressing intraspecific attack had no significant effect on sunflower seed acceptance latencies $[t(19)=1.02$, $\mathrm{p}>.1]$. These data are shown in Table 1 .

\section{Reinforcing Value of AIS}

A scatter plot of pedalpress rates vs. current intensity at 16 sites in six subjects shows a significant decline from above to below baseline rates as AIS current increases (Figure 2 ). This graph clearly suggests that AIS is neither necessarily rewarding nor necessarily aversive.

\section{Supplementary Sex Test}

The four subjects tested exhibited mounting and pelvic thrusting on the hormonally primed, receptive female. Mean latencies for pelvic thrusting were .99 , .52 , and $.28 \mathrm{~min}$ on the first, second, and fourth (unstimulated) trials, respectively. On the third (stimulated) trial, two animals whose mean AIS level was $45 \mu \mathrm{A}$ had mount and thrust latencies of $.60 \mathrm{~min}$; the sexual behavior of the other two animals, whose mean AIS level was $92 \mu \mathrm{A}$, was completely inhibited for the full 2-min trial.

\section{DISCUSSION}

We have extended earlier demonstrations of septal inhibition of predatory and defensive aggression in rats, cats, and monkeys by showing that septal stimulation also inhibits intraspecific aggression in hamsters. To insure that this inhibition was not a consequence of response competition between attack and some stimulation-produced behavior, we were generally able to use currents below those which elicited an overt response. During AIS, subjects often approached, sniffed, and followed targets, behaviors which ordinarily precede attack. Thus, stimulation did not interfere with the sensory, motor, or motivational processes involved in the localization, tracking, and investigation of the target. On three occasions, subjects adopted aggressive postures during AIS, suggesting that, within the usual sequence of agonistic behavior, the "consummatory response" of overt attack was the most susceptible to inhibition.

The lack of significant AIS effects on sunflower seed acceptance, another "oral" behavior, further suggests that this inhibition was relatively specific. Similarly specific effects on aggression, but not eating, have been found with septal inhibition of muricidal (Potegal, Gibbons, \& Glusman, 1980) and defensive behavior (Brayley \& Albert, 1977) in rats. The particular features of these behaviors which render them differentially susceptible to inhibition (e.g., latency, nature of the goal object, response complexity, and topography, etc.) remain to be investigated. Additional support for the specificity hypothesis also comes from our observation that low-current AIS that completely suppressed aggression in two animals had a negligible effect on sexual behavior. However, higher level AIS in two other animals suppressed sexual behavior. These results indicate that, at sufficiently low current levels, it is possible to selectively inhibit aggression without necessarily affecting other behaviors (Brayley \& Albert, 1977).

This proposal of inhibition specificity would be strengthened by a demonstration that AIS was not producing electrographic seizure activity. Electrographic recordings were, in fact, made on our subjects before and after stimulation. While these recordings did not suggest the occurrence of seizure activity, the quality of recordings possible from our movable electrode proved inadequate for a conclusive demonstration. Such recordings should be repeated with rigid, bipolar septal AIS electrodes.

The "carryover" effect, in which AIS on one trial reduced the probability of aggression on subsequent nonstimulated trials, has also been observed in cats (Adamec, 1976). Carryover effects in the inhibition of lordosis in female hamsters by septal stimulation have also been reported (Zasorin, Malsbury, \& Pfaff, 1975). Shipley and Kolb's (1977) report that septal lesions in hamsters prolong the duration of shock-facilitated attack seems to be at least superficially complementary to our observation that septal stimulation is followed by a prolonged inhibition of attack.

Reports that aggression-inhibitory brain stimulation may be positively reinforcing (Plotnick, Mir, \& Delgado, 1971), negatively reinforcing (Chaurand, Vergnes, \& Karli, 1974), or neutral (Brayley \& Albert, 1977) suggest that the aggression-inhibitory aspect of such stimulation is independent of its reward value. Such independence is confirmed by our own finding that the reinforcing value of AIS appears to be a function of current intensity; AIS below $55 \mu \mathrm{A}$ produced above-baseline pedalpress rates, while higher current AIS reduced pedalpressing to below baseline rates. Strictly speaking, these data do not form a standard rate-intensity function, but they do bear a resemblance to the descending phase of such functions (cf. Atrens, 1970, particularly the slope of the rate-intensity function for the tuberculopiriform tract, Figure 2).

Figure 1 indicates that aggression inhibition was obtained from 11 of 12 points within the septum but from only 2 of 4 points lateral or dorsal to the septum and only 5 of 9 points ventral to the anterior commissure. A V-shaped profile of inhibition thresholds, seen in three of four coronal planes, has its apex within the septum. These observations suggest that 
stimulation of structure(s) within the septum is sufficient to inhibit intraspecific aggression. Inhibition threshold values ranged from 30 to $130 \mu \mathrm{A}$; these values correspond to current spread distances of .2$.5 \mathrm{~mm}$ for cell bodies and .3-1.1 mm for axons, according to Ranck (1975, Figure 1). Given this range of current spread distance and the small size of the hamster septum (e.g., the vertical distance between the ventral edge of the corpus callosum and the dorsal edge of the anterior commissure in section A4.8 of Figure 1 is approximately $1.5 \mathrm{~mm}$ ), it is probable that a substantial portion ô septal region tissue was being stimulated on most trials. However, we conjecture that it is not the volume of septal tissue being stimulated that is important, but rather certain structures within the septal region which are essential to the inhibitory effects. The basis for this conjecture is the relatively close match between our inhibition profiles and Case II of Wise's (1972, Figure 2) theoretical analysis of threshold profiles generated by movable electrodes. The profile of his Case II is generated by a "neural system" around which a stimulation field, large relative to the "system," is descending. Among the basal septal region structures that might make up the "system" are the anterior commissure and the bed nucleus of the stria terminalis, both of which project to the amygdala, a known aggression inhibitory area in rodents (Vergnes \& Karli, 1969). Evidence for a contribution of the nucleus acumbens to intraspecific aggression in rodents (Albert \& Wong, 1978) suggests that this structure should be considered too.

Two of the electrode tracks extended into the preoptic area; aggression inhibition was obtained from the more lateral of the two. However, septal and preoptic inhibition of aggression may involve somewhat different mechanisms. The reinforcing or aversive value of septal region AIS was a function of current level, while preoptic AIS seemed to be more consistently aversive regardless of current level. Similarly, a difference apparently exists with regard to suppression of lordosis in female hamsters produced by septal or preoptic stimulation. While stimulation of both areas can suppress lordosis, during septal stimulation "the animals remained relatively quiet" (Zasorin et al., 1975, p. 596), while during preoptic-anterior hypothalamic stimulation "most females showing lordosis suppression increased their locomotor activity"' (Malsbury, Pfaff, \& Malsbury, 1980, p. 275).

What might be the behavioral significance of the aggression inhibitory effects of septal region activation? According to field studies, the adult hamster is a territorial isolate (see Johnston, 1975, for review); such animals are usually intolerant of conspecifics. Given that aggression may be a routine accompaniment of intraspecific encounters in the hamster, one possibility is that such a specific aggression-inhibitory system might be involved with the suppression of aggressive responses as a prerequisite for successful mating. In laboratory studies, at least, male hamsters are far less aggressive toward estrous female hamsters than they are toward other male hamsters (e.g., Frisk, Wagner, \& Kusewitt, 1977). Some support for the conjecture that the septum may be involved in this effect comes from our observation that male hamsters with lesions of the anteroventral septum systematically attack, rather than copulate with, hormonally primed receptive females (Potegal, Blau, \& Glusman, 1981).

\section{REFERENCE NOTE}

1. Knigge, K. M., \& Joseph, S. A. A stereotaxic atlas of the brain of the golden hamster, Mesocricetus auratus. Cincinnati: Department of Anatomy, University of Cincinnati, 1966.

\section{REFERENCES}

Adamec, R. E. Hypothalamic and extrahypothalamic substrates of predatory attack. Suppression and the influence of hunger. Brain Research, 1976, 106, 57-69.

Albert, D. J., \& Wong, R. C. K. Hyperreactivity, muricide, and intraspecific aggression in the rat produced by infusion of local anesthetic into the lateral septum or surrounding areas. Journal of Comparative and Physiological Psychology, 1978, 92, 1062 1073.

Atrens, D. M. Reinforcing and emotional consequences of electrical self-stimulation of the subcortical limbic forebrain. Physiology \& Behavior, 1970, 5, 1461-1471.

Brayley, K. N., \& Albert, D. J. Suppression of VMH-lesioninduced reactivity and aggressiveness in the rat by stimulation of lateral septum, but not medial septum or cingulate cortex. Journal of Comparative and Physiological Psychology, 1977, 91, 290-299.

Chaurand, J. P., Vergnes, M., \& Karli, P. Declenchement de conduites agressives par stimulation electrique du tegmentum ventral du mesencephale chez le rat. Physiology \& Behavior, 1974, 12, 771-778.

Floody, O. R., \& Pfaff, D. W. Aggressive behavior in female hamsters: The hormonal basis for fluctuations in female aggressiveness correlated with estrous state. Journal of Comparative and Physiological Psychology, 1977, 91, 443-464.

Frisk, C. S., Wagner, J. E., \& KusewitT, D. F. Unusual aggressive behavior in the male golden hamster. Laboratory Animal Science, 1977, 27, 682-684.

Grant, E. C., \& Mackintosh, J. H. A description of the social postures of some laboratory rodents. Behaviour, 1963, 21, 246-259.

JAnzen, W. B., \& Bunnell, B. N. Septal lesions and the recovery of function in the juvenile hamster. Physiology \& Behavior, $1976,16,445-452$.

Jasper, H. H., \& Ajmone-Marsan, C. A stereotaxic atlas of the diencephalon of the cat. Ottawa: National Research Council of Canada, 1954.

Johnson, D. A., Poplawsky, A., \& Bieliauskas, L. Alterations of social behavior in rats and hamsters following lesions of the septal forebrain. Psychonomic Science, 1972, 26, 19-20.

Johnston, R. E. Scent marking by male golden hamsters (Mesocricetus auratus): III. Behavior in a seminatural environment. Zeitschrift für Tierpsychologie, 1975, 37, 213-221.

Malsbury, C. W., Pfaff, D. W., \& Malsbury, A. M. Suppression of sexual receptivity in the female hamster: Neuroanatomical projections from preoptic and anterior hypothalamic electrode sites. Brain Research, 1980, 181, 267-284.

Plotnick, R., Mir, D., \& Delgado, J. M. R. Aggression, noxiousness and brain stimulation in unrestrained rhesus mon- 
keys. In B. Eleftheriou \& J. Scott (Eds.), The physiology of aggression and defeat. New York: Plenum Press, 1971.

Potegal, M., Blau, A., Black, M., \& Glusman, M. A technique for the study of intraspecific aggression in the golden hamster under conditions of reduced target variability. Psychological Record, 1980, 30, 191-200.

Potegal, M., Blau, A., \& Glusman, M. Effects of anteroventral septal lesions on intraspecific aggression in male hamsters. Physiology \& Behavior, 1981, 26, 407-412.

Potegal, M., Gibbons, J., \& Glusman, M. Inhibition of muricide by septal stimulation in rats. Physiology \& Behavior, 1980, 24, 863-867.

RANCK, J. B. Which elements are excited in electrical stimulation of mammalian central nervous system: A review. Brain Research, 1975, 98, 417-440.

Rubinstein, E. H., \& Delgado, J. M. R. Inhibition induced by forebrain stimulation in the monkey. American Journal of Physiology, 1963, 205, 941-948.

SHIPLEY, J. E., \& KolB, B. Neural correlates of species-typical behavior in the Syrian golden hamster. Journal of Comparative and Physiological Psychology, 1977, 91, 1056-1073.

Siegel, A., \& Skog, D. Effects of electrical stimulation of the septum upon attack behavior elicited from the hypothalamus in the cat. Brain Research, 1970, 23, 371-380.

Sierra, G., Acuna, C., Otero, J., \& Dominguez, R. Simultaneous stimulation of limbic system structures. Brain Research, 1972, 47, 113-125.

Sodetz, F. J., \& Bunnell, B. N. Septal ablation and the social behavior of the golden hamster. Physiology \& Behavior, 1970, 5, 79-88.

Vergnes, M., \& Karli, P. Effets de la stimulation de l'hypothalamus lateral, de l'amygdale et de l'hippocampe sur le comportement d'agression interspecifique rat souris. Physiology \& Behavior, 1969, 4, 889-894.

WISE, R. A. Spread of current from monopolar stimulation of the lateral hypothalamus. American Journal of Physiology, $1972,223,545-548$.

Zasorin, N. L., Malsbury, C. W., \& Pfaff, D. W. Suppression of lordosis in the hormone-primed female hamster by electrical stimulation of the septal area. Physiology \& Behavior, 1975, 14, 595-600.

(Received for publication December 20, 1980; revision accepted April 17, 1981.) 